
MARGRET TUCHSCHERER

\title{
Die Robustheit von BLUP und EBLUP
}

\begin{abstract}
Summary
Title of the paper: The Robustness of BLUP and EBLUP

The expectations of BLP and BLUP used as estimator for fixed effects and as predictor for random effects were investigated concerning the property 'unbiasedness'. Different aspects of robustness research on prediction procedures are discussed. The robustness of BLUP and EBLUP was investigated with respect to the distribution of the random components of the model, the degree of balancedness of the experimental design, influence of the variance components ratio and different variance component estimators used in EBLUP(2) on the bases of a computer simulation in the random one-way model. Criteria used for the evaluation were the mean squared error (MSE) and the selection gain. Besides, an idea of the overestimation of the accuracy of EBLUP by the naive MSE approximation based on the MSE formulas of BLUP with variance component estimations instead of unknown parameters is given.
\end{abstract}

Key words: mixed linear model, BLUP, EBLUP, robustness, MSE of prediction, selection gain

\section{Zusammenfassung}

Die Erwartungswerte der BLP und der BLUP bei Verwendung als Schätzung für die festen Effekte als auch als Vorhersage für die zufälligen Effekte werden bezüglich der Eigenschaft „Erwartungstreue" untersucht. Unterschiedliche Aspekte der Robustheitsforschung an Vorhersageverfahren werden diskutiert. Die Robustheit von BLUP und EBLUP wird bezüglich der Verteilung der zufälligen Komponenten des Modells, des Balanciertheitsgrades des Versuchsplanes, des Einflusses des Varianzkomponentenverhältnisses und unterschiedlicher Varianzkomponentenschätzer, die in $\operatorname{EBLUP}(2)$ verwendet werden, untersucht. Als Kriterien für die Bewertung wurden der mittlere quadratische Fehler (MSE) der Vorhersage und der Zuchtfortschritt gewählt. Außerdem wird eine Vorstellung über die Genauigkeitsüberschätzung der EBLUP durch die naive MSE-Approximation, die auf den Formeln des MSE basiert, bei denen die Varianzkomponenten durch entsprechende Schätzwerte ersetzt wurden, gegeben.

Schlüsselwörter: gemischtes lineares Modell, BLUP, EBLUP, Robustheit, Vorhersage-MSE, Zuchtfortschritt

1 . Einleitung

Mit Hilfe verschiedener Verfahren der Zuchtwertvorhersage werden Zuchtobjekte in eine Reihenfolge gebracht und anschließend selektiert. Im Rahmen der gerichteten Selektion geschieht das durch Stutzungsselektion. Die theoretische Basis der Stutzungsselektion bildet der grundlegende Satz der Selektionstheorie von COCHRAN (1951). Dieser Satz fordert insbesondere die Existenz des bedingten Erwartungswertes analog zur Theorie der Regression Modell II. Die Stutzungsselektion sollte, wenn möglich, nach dem bedingten Erwartungswert erfolgen. Dieser bedingte Erwartungswert stellt im Rahmen der Vorhersage gerade die beste Vorhersage (BP) dar. Zur Selektion kann aber auch jede monotone Transformation der BP verwendet werden, da 
dadurch die Reihenfolge der Zuchtobjekte nicht verändert wird und damit auch der Absolutwert der korrelierten Selektionsdifferenz (Selektionserfolg im Zuchtziel) nicht verkleinert wird. Leider ist die BP im allgemeinen nicht bekannt. Unterstellt man jedoch Normalverteilung für alle Zufallsvariablen des Modells, so gilt ein lineares Modell und die sogenannte beste lineare Vorhersage (BLP) stellt auch die BP dar. Im Falle der Normalverteilung kann also nach der BLP selektiert werden, ohne daß ein Verlust an Selektionserfolg eintritt. Das ist für andere Verteilungen nicht in dieser Form nachgewiesen. Da in der BLP neben den Varianzkomponenten auch die festen Effekte als bekannt vorausgesetzt werden, hat man, um die Anzahl der als bekannt vorauszusetzenden Parameter auf die Anzahl der Varianzkomponenten zu reduzieren, die sogenannte beste lineare erwartungstreue Vorhersage (BLUP) entwickelt. Die grundlegenden Eigenschaften der BLP und BLUP werden in den Sätzen von HENDERSON $(1963,1973,1975)$ klargelegt. Sowohl die BLP als auch die BLUP sind im Rahmen des gemischten linearen Modells erwartungstreu. Die BLP liegt in der Klasse $\left\{c+b^{\prime} \underline{Y}\right\}$, wenn mit $\mathrm{c}$ eine Konstante, mit b ein Vektor von Konstanten und mit $\underline{Y}$ das Modell für den Vektor aller Beobachtungen bezeichnet werden. Dagegen liegt die BLUP in der Klasse $\left\{b^{\prime} \underline{y}\right\} \cap\{E(\underline{w})=E[F(\underline{Y})]\}$, wenn mit $\underline{w}$ die vorherzusagende Zufallsgröße und mit $\mathrm{F}(\underline{\mathrm{Y}})$ irgendeine Funktion der Beobachtungswerte bezeichnet werden. Treten im gemischten linearen Modell feste Effekte oder mindestens ein von Null verschiedener Mittelwert auf, so sind beide Vorhersagen verschieden, obwohl sie beide linear und erwartungstreu sind. Selbst bei Unterstellung von Normalverteilung, die bei der Ableitung nicht allgemein unterstellt wird, sind BLP und BLUP verschiedene Vorhersagen und können zu verschiedenen Rangfolgen der Zuchtobjekte führen. Der Selektionserfolg nach BLUP ist auf jeden Fall kleiner oder gleich dem Selektionserfolg nach BLP.

Sowohl die BLP als auch die BLUP sind nicht anwendungsbereit, da die benötigten Parameter als unbekannt angenommen werden müssen. Anwendungsbereit sind nur verschiedene Formen der geschätzten BLP oder BLUP (mit EBLP bzw. EBLUP bezeichnet). Setzt man für die Parameter Konstanten ein, so erhält man EBLP(1) und EBLUP(1). Beide sind lineare erwartungstreue Vorhersagen wie vorher, haben nun aber nicht mehr die Eigenschaft, beste in ihrer Klasse zu sein, es sei denn, man hat die tatsächlichen Parameter getroffen. Andererseits können die unbekannten Parameter auch mit $\underline{Y}$ geschätzt werden. In diesem Falle erhält man EBLP(2) - bzw. EBLUP(2)Verfahren. Dabei gibt es verschiedene Varianten je nach dem zur Schätzung der Parameter verwendeten Verfahren. EBLP(2) und EBLUP(2) liegen nicht mehr in der Klasse der linearen Vorhersagen.

BLP und BLUP werden unter sehr allgemeiner Verteilungsannahme derart abgeleitet, daß sie den mittleren quadratischen Fehler (MSE) der Vorhersage in ihrer Klasse minimieren. Da sie beide bezüglich der Vorhersage erwartungstreu sind, genügt zu ihrer Beurteilung die Varianz des Vorhersagefehlers. Die Wurzel aus dieser Varianz wird z. B. in SAS (proc mixed) als Vorhersagegenauigkeit von BLUP angegeben. Die weiteren Momente der Verteilung spielen hier keine Rolle.

EBLP(1) und EBLUP(1) sind immer bezüglich der Vorhersage erwartungstreu, können 
aber bezüglich der Vorhersagegenauigkeit nur dann beurteilt werden, wenn bekannt ist, in welcher Weise die vorgegebenen Konstanten für die Parameter von diesen abweichen. Liegen derartige Informationen vor, so kann die Vorhersagegenauigkeit mit Hilfe der Varianz des Vorhersagefehlers beurteilt werden.

Anders verhält es sich mit EBLP(2) und EBLUP(2). Diese Vorhersagen müssen nicht mehr erwartungstreu sein. In diesem Falle sollte zur Beurteilung der MSE herangezogen werden, und dieser kann von den weiteren Momenten der Verteilung abhängen, insbesondere von Schiefe und Exzeß, aber auch noch von weiteren Parametern der Verteilung. Die Berechnung des MSE kann sich jedoch problematisch gestalten. Aus diesem Grunde werden häufig 'naive' Approximationen für den MSE derart angegeben, daß man die Schätzwerte für die Varianzkomponenten, die auf der Basis von $\underline{Y}$ gewonnen wurden, in die Varianzformeln des Vorhersagefehlers einsetzt (z.B. proc mixed in SAS). Welchen Fehler begeht man bei einer derartigen Genauigkeitsangabe?

Natürlich haben EBLP(2) und EBLUP(2) nun auch keinesfalls die guten Eigenschaften von BLP bzw. BLUP bei der Selektion. Die Aufklärung derartiger und anderer Probleme gehört zu den Aufgaben von Robustheitsuntersuchungen. Es gibt sehr verschiedene Aspekte der Robustheit von Vorhersageverfahren, einige davon werden hier betrachtet.

\section{Erwartungstreue von BLP und BLUP bezüglich Vorhersage und Schätzung}

Jede beliebige Funktion der Beobachtungen kann auch als Schätzfunktion verwendet werden. Daher können BLP und BLUP auch als Schätzfunktion genutzt werden, wobei natürlich festzulegen ist, für welche feste Größe. Im gemischten linearen Modell gibt es keinen festen Zuchtwert, daher können in diesem Modell BLP oder BLUP auch nicht als Zuchtwertschätzung verwendet werden. Soll das dennoch geschehen, so muß der Zuchtwert als feste Größe definiert werden. Das gemischte lineare Modell hat die Form

$$
\underline{\mathrm{Y}}=\mathrm{X} \beta+\mathrm{Z} \underline{\mathrm{u}}+\underline{\mathrm{e}} .
$$

Die Vorhersagen beziehen sich hier auf

$$
\underline{\mathrm{w}}=\mathrm{k}^{\prime} \beta+\mathrm{m}^{\prime} \underline{\mathrm{u}}
$$

gemäß HENDERSON (1975). Es gilt

und damit

$$
\mathrm{E}(\underline{\mathrm{Y}})=\mathrm{X} \beta, \quad \mathrm{E}(\mathrm{Z} \underline{\mathrm{u}})=\mathrm{E}(\underline{\mathrm{e}})=0, \quad \mathrm{~V}(\underline{\underline{\mathrm{u}}} \underline{\mathrm{e}})=\left(\begin{array}{cc}
\mathrm{G} & 0 \\
0 & \mathrm{R}
\end{array}\right) \sigma^{2}
$$

$$
\mathrm{V}(\underline{\mathrm{Y}})=\sigma^{2}(\mathrm{R}+\mathrm{ZGZ})=\sigma^{2} \mathrm{~V}, \quad 0<|\mathrm{R}|<\infty ; 0<|\mathrm{G}|<\infty .
$$

Die beste lineare Vorhersage (BLP) hat die Form

$$
\underline{\hat{w}}=k^{\prime} \beta+m^{\prime} G Z V^{-1}(\underline{Y}-X \beta) \text {. }
$$

Es sei nun $u$ eine Realisation von $\underline{u}$, dann kann $w=k^{\prime} \beta+m^{\prime} u$ als Realisation von $\underline{w}$ angesehen werden. Diese feste Größe kann durch die BLP geschätzt werden. Es gilt

$$
\mathrm{E}(\underline{\hat{w}} \mid \underline{w}=w)=k^{\prime} \beta+m^{\prime} G Z^{\prime} V^{-1}(X \beta+Z u-X \beta)=k^{\prime} \beta+m^{\prime} G Z^{\prime} V^{-1} Z u \text {. }
$$

Wenn $\mathrm{GZ}^{\prime} \mathrm{V}^{-1} \mathrm{Z}$ nicht die Einheitsmatrix ist, dann ist 
$E(\underline{\hat{w}} \mid \underline{w}=w) \neq k^{\prime} \beta+m^{\prime} u$

und damit nicht erwartungstreu. An einem einfachen Beispiel soll dieses Ergebnis demonstriert werden. Es sei eine Zufallsstichprobe von q Bullen gegeben. Jeder dieser Bullen sei mit einer Zufallsstichprobe von Kühen gepaart, so daß je Bulle $\mathrm{n}$ Nachkommen entstanden sind. Für die $\underline{y}_{i j}(i=1, \ldots, q ; j=1, \ldots, n)$ gelte das Modell

$$
\underline{y}_{i j}=\mu+\frac{1}{2} \underline{g}_{s i}+\underline{e}_{i j},
$$

wobei $\underline{\mathrm{g}}_{\mathrm{si}}$ den Zuchtwert des i-ten Bullen symbolisiert. Dann gilt nach (1) mit $\hat{\underline{w}}_{\mathrm{i}}=\underline{\mathrm{g}}_{\mathrm{si}}$

$$
\hat{\mathrm{g}}_{\mathrm{si}}=2\left(\sum_{\mathrm{j}=1}^{\mathrm{n}}\left(\underline{\mathrm{y}}_{\mathrm{ij}}-\mu\right) \frac{\frac{1}{4} \mathrm{~h}^{2}}{1+\frac{1}{4}(\mathrm{n}-1) \mathrm{h}^{2}}\right)
$$

und damit

$$
\mathrm{E}\left(\underline{\mathrm{g}}_{\mathrm{si}} \mid \underline{\mathrm{g}}_{\mathrm{si}}=\mathrm{g}_{\mathrm{si}}\right)=\frac{\frac{1}{4} \mathrm{nh}^{2}}{1+\frac{1}{4}(\mathrm{n}-1) \mathrm{h}^{2}} \mathrm{~g}_{\mathrm{si}} \text {. }
$$

$\hat{\mathrm{g}}_{\mathrm{si}}$ ist in diesem Sinne keine erwartungstreue Schätzung für $\mathrm{g}_{\mathrm{si}}$, jedoch asymptotisch erwartungstreu. Zur Beurteilung als Schätzung muß also der MSE herangezogen werden, nicht etwa nur die Varianz. Analog kann bei der Beurteilung von BLUP als Schätzung für w vorgegangen werden. Es gilt

$$
\underline{\widetilde{w}}=k^{\prime} \underline{\hat{\beta}}+m^{\prime} \underline{\tilde{u}}
$$

mit

$$
\begin{aligned}
& \underline{\tilde{u}}=G Z^{\prime} V^{-1}(\underline{Y}-X \underline{\hat{\beta}}), \\
& \underline{\hat{\beta}}=\left(X^{\prime} V^{-1} X\right)^{-} X^{\prime} V^{-1} V^{-1} \underline{Y}=B \underline{Y} .
\end{aligned}
$$

HENDERSON (1973) zeigte, daß die Lösungen $\underline{\hat{\beta}}$ und $\underline{\tilde{u}}$ des sogenannten 'mixed model'- Gleichungssystems

$$
\left(\begin{array}{cc}
X^{\prime} R^{-1} X & X^{\prime} R^{-1} Z \\
Z^{\prime} R^{-1} X & Z^{\prime} R^{-1} Z+G^{-1}
\end{array}\right)\left(\begin{array}{l}
\hat{\beta} \\
\tilde{\tilde{u}}
\end{array}\right)=\left(\begin{array}{c}
X^{\prime} R^{-1} \underline{Y} \\
Z^{\prime} R^{-1} \underline{Y}
\end{array}\right),
$$

die man ohne die numerisch aufwendige Invertierung der Matrix $\mathrm{V}=\mathrm{ZGZ}+\mathrm{R}$ erhält, mit (4)

identisch sind.

Unter der Bedingung fester Zuchtwerte gilt

$$
\underline{\mathrm{Y}}_{\underline{\mathrm{u}}=\mathrm{u}}=\mathrm{X} \beta+\mathrm{Zu}+\underline{\mathrm{e}} \text {. }
$$

Setzt man (4) und (5) in (3) ein, so folgt

$$
\underline{\widetilde{w}}_{\underline{\underline{u}}=u}=k^{\prime} B(X \beta+Z u+\underline{e})+m^{\prime} G Z^{\prime} V^{-1}(X \beta+Z u+\underline{e}-X B[X \beta+Z u+\underline{e}]
$$

und man erhält

$$
\begin{aligned}
\mathrm{E}(\underline{\hat{\mathrm{w}}} \mid \underline{\mathrm{u}}=\mathrm{u})= & \mathrm{k}^{\prime} \mathrm{B}(\mathrm{X} \beta+\mathrm{Zu})+\mathrm{m}^{\prime} G Z^{\prime} \mathrm{V}^{-1}(X \beta+Z u-X B[X \beta+Z u]) \\
& =\mathrm{k}^{\prime} \beta+\left[\mathrm{k}^{\prime} B+\mathrm{m}^{\prime} G Z^{\prime} \mathrm{V}^{-1}(I-X \beta)\right] Z u=k^{\prime} \beta+Q^{\prime} u .
\end{aligned}
$$


Gewöhnlich gilt $Q^{\prime} \neq m^{\prime}$ und damit ist BLUP keine erwartungstreue Schätzung. Auch hier muß der MSE zur Beurteilung herangezogen werden.

3. Verschiedene Konzepte der Robustheitsforschung

Die Robustheit einer Vorhersage oder Schätzung kann in verschiedener Hinsicht untersucht werden. Es gibt folgende Robustheitsrichtungen, wobei alle als Abweichung gegenüber dem unterstellten Modell einschließlich der Voraussetzungen und Nebenbedingungen definiert sind.

\subsection{Die Robustheit gegenüber Abweichungen von der unterstellten Verteilung}

Da sowohl bei der BLUP als auch bei der BLP keine spezielle Verteilung unterstellt wurde, sind beide in dieser Hinsicht nicht zu untersuchen. Robustheitsuntersuchungen sind überflüssig. Das gleiche gilt auch für die EBLUP(1). Die EBLUP(2) jedoch ist keine lineare Funktion von $\underline{Y}$ und damit sind bei der Beurteilung über den MSE auch höhere Momente der Verteilung von Bedeutung. Der MSE ist eine Funktion nicht nur von den beiden ersten Momenten.

\subsection{Die Robustheit gegenüber Ausreißern}

Ausreißer beeinflussen alle Momente der Verteilung. Eine Untersuchung sowohl von BLP, BLUP als auch EBLUP(1) und EBLUP(2) ist sinnvoll, wird aber hier nicht vorgenommen.

Die Behandlung von Ausreißern in der Zuchtwertvorhersage ist ein offenes Problem. Es erscheint jedoch sinnvoll, die bekannte Theorie von HUBER (1981) auch auf die Zuchtwertvorhersage anzuwenden, um Ausreißer besser beurteilen zu können und um deren Einfluß bei der praktischen Zuchtwertvorhersage zu verringern.

Alle Zuchtwertvorhersageverfahren hängen vom verwendeten Modell ab. So kann es zu zum Teil erheblichen Verzerrungen der Zuchtwertvorhersage führen, wenn Einflußgrößen nicht oder in falscher Form berücksichtigt werden. Gerade bei der praktischen Zuchtwertvorhersage spielt diese eine zentrale Rolle. Entsprechende Untersuchungen zur Modellrobustheit wie z.B. über geeignete Splitting der Daten oder Computersimulation sind also durchaus angebracht, werden hier aber nicht weiter betrachtet.

Die Robustheit gegenüber falscher Parameterwahl

Bei der EBLUP(1) sind die Parameter vorzugeben, daher ist eine Untersuchung der EBLUP(1) in dieser Hinsicht sinnvoll. HENDERSON (1975) analysierte den Einfluß unkorrekter Parameter auf die Erwartungstreue und die Varianz der Vorhersage in 
EBLUP(1).

Sind in (6) $G^{*}=G$ und $R^{*}=R$, so ist $\underline{\widetilde{w}}=k^{\prime} \hat{\beta}+m^{\prime} \underline{\tilde{u}}$ eine BLUP von $\underline{w}=k^{\prime} \beta+m^{\prime} \underline{u}$ wenn $\hat{\hat{\beta}}=\hat{\beta}^{*}$ und $\underline{\tilde{u}}=\underline{\tilde{u}}^{*}$ Lösungen des Gleichungssystems

$$
\left(\begin{array}{cc}
X^{\prime} R^{*-1} X & X^{\prime} R^{*-1} Z \\
Z^{\prime} R^{*-1} X & Z^{\prime} R^{*-1} Z+G^{*-1}
\end{array}\right)\left(\begin{array}{l}
\hat{\beta}^{*} \\
\tilde{\widetilde{u}}^{*}
\end{array}\right)=\left(\begin{array}{c}
X^{\prime} R^{*-1} \underline{Y} \\
Z^{\prime} R^{*-1} \underline{Y}
\end{array}\right)
$$

sind (HENDERSON, 1963). Verwendet man anstelle bekannter Matrizen G und / oder R Schätzwertmatrizen $\mathrm{G}^{*}$ und / oder $\mathrm{R}^{*}$, die sich in der Regel von $\mathrm{G}$ bzw. $\mathrm{R}$ unterscheiden $\left(\mathrm{G}^{*} \neq \mathrm{G}\right.$ und / oder $\left.\mathrm{R}^{*} \neq \mathrm{R}\right)$, so ist die Vorhersage EBLUP(1) $\underline{\widetilde{\mathrm{w}}}{ }^{*}=\mathrm{k}^{\prime} \underline{\hat{\beta}}^{*}+\mathrm{m}^{\prime} \underline{\widetilde{u}}^{*}$ mit den Lösungen $\hat{\beta}^{*}$ und $\underline{\widetilde{u}}^{*}$ des Gleichungssystems (6) keine BLUP von $\underline{w}=k^{\prime} \beta+m^{\prime} \underline{u}$, denn bei Zuchtwertvorhersage ohne Selektion kann man leicht zeigen, da $\beta \mathrm{E}(\underline{\underline{w}})=\mathrm{E}(\underline{\widetilde{w}})=\mathrm{E}\left(\underline{\underline{w}}^{*}\right)$ gilt, d.h. die Erwartungstreue bleibt erhalten, woraus wiederum trivialer Weise folgt, daß

$\operatorname{MSE}(\underline{\widetilde{w}})=V(\underline{\widetilde{w}}-\underline{w})<\operatorname{MSE}\left(\underline{\widetilde{w}}^{*}\right)=V\left(\underline{\widetilde{w}}^{*}-\underline{w}\right)$. Somit ist offensichtlich, daß bei unkorrektem G und / oder $\mathrm{R}$ ein erhöhter Vorhersagefehler auftritt. Als Genauigkeitsmaß für die EBLUP(1) $\underline{\widetilde{w}}^{*}$ ist die Varianz des Vorhersagefehlers $\mathrm{V}\left(\underline{\tilde{w}}^{*}-\underline{w}\right)$ nur dann brauchbar, wenn mit $G^{*}$ bzw. $R^{*}$ auch die Differenz zu G bzw. $R$ quantifiziert werden kann.

Bei der EBLUP(2) sind keine Parameter zu wählen. Sie ist in dieser Hinsicht also auch nicht $\mathrm{zu}$ untersuchen.

Die Robustheit gegenüber dem Versuchsplan

Der Versuchsplan hat einen ganz erheblichen Einfluß auf die Güte aller Verfahren. Balancierte Versuche sind bei gleichem Versuchsumfang wesentlich effektiver als unbalancierte. Es lohnt sich immer, bevor man sich an die Verbesserung der Zuchtwertvorhersage macht, die Möglichkeit zu prüfen, inwieweit der Versuchsplan (Zuchtorganisation) optimaler gestaltet werden kann.

4. Die Robustheit von EBLUP(2) als Vorhersage beziglich der Verteilung, dem Grad der Balanciertheit und dem Verhältnis der beiden Varianzkomponenten im Modell II einer einfachen Varianzanalyse

4.1

Modell und Vorhersageverfahren

Das Modell II der einfachen Varianzanalyse nach EISENHART (1947) hat die Form

$$
\underline{y}_{i j}=\mu+\underline{u}_{i}+\underline{e}_{i j} \quad\left(i=1, \ldots, a ; j=1, \ldots, n_{i}\right)
$$

mit

$$
\begin{aligned}
& E\left(\underline{u}_{i}\right)=E\left(\underline{e}_{i j}\right)=0, E\left(\underline{y}_{i j}\right)=\mu, \\
& V\left(\underline{u}_{i}\right)=\sigma_{u}^{2} \quad, V\left(\underline{e}_{i j}\right)=\sigma_{e}^{2}, \quad \operatorname{cov}\left(\underline{u}_{i}, \underline{e}_{i j}\right)=0 .
\end{aligned}
$$


Nach (3) erhält man die BLUP aus

mit

$$
\underline{\tilde{u}}_{i}=\frac{\sigma_{u}^{2}}{\sigma_{e}^{2}+n_{i} \sigma_{u}^{2}} \cdot \sum_{j=1}^{n_{i}}\left(\underline{y}_{i j}-\underline{\hat{\mu}}\right)
$$

$$
\underline{\mu}=\sum_{i=1}^{a} \frac{\underline{y}_{i}}{\sigma_{e}^{2}+n_{i} \sigma_{u}^{2}} / \sum_{i=1}^{a} \frac{n_{i}}{\sigma_{e}^{2}+n_{i} \sigma_{u}^{2}} .
$$

Um (7) anwenden zu können benötigt man Schätzwerte für die beiden Varianzkomponenten. Im Falle der EBLUP(2) werden hier auf der Basis der $\underline{y}_{i j}$ die Varianzkomponentenschätzverfahren

(1) ANOVACOR

ANalysis Of VAriance estimation, (COR: negative Varianzkomponentenschätzwerte auf 0 korrigiert)

(2) $\mathrm{ML}$ Maximum Likelihood estimation

(3) REML Restricted Maximum Likelihood estimation

(4) I-MINQUECOR MInimum Norm Quadratic Unbiased Estimation (COR: negative Varianzkomponentenschätzwerte auf 0 korrigiert; I: iterativ)

genutzt. Alle hier verwendeten Schätzfunktionen sind Abbildungen des Stichprobenraumes in den Parameterraum, d. h., es werden nur nichtnegative Schätzwerte zugelassen. Die daraus resultierenden vier Varianten der EBLUP(2), hier mit $\operatorname{EBLUP}(2,1), \operatorname{EBLUP}(2,2), \operatorname{EBLUP}(2,3)$ bzw. $\operatorname{EBLUP}(2,4)$ bezeichnet, sollen nun bezüglich ihre Güte untersucht und mit der BLUP verglichen werden. Die Güte dieser Vorhersagen soll mit Hilfe des MSE der Vorhersagefehler dieser Verfahren in Abhängigkeit von der Verteilung der $\underline{u}_{\mathrm{i}}$ und $\underline{\mathrm{e}}_{\mathrm{ij}}$, dem Grad der Balanciertheit der zugrundeliegenden Versuchspläne $\left(\mathrm{N}, \mathrm{a}, \mathrm{n}_{1}, \mathrm{n}_{2}, \ldots, \mathrm{n}_{\mathrm{a}}\right)$ bei einem Gesamtumfang $\mathrm{N}$ und dem Verhältnis der beiden Varianzkomponenten $\sigma_{\mathrm{u}}^{2} / \sigma_{\mathrm{e}}^{2}$ untersucht werden. Da die analytische Berechnung des MSE erhebliche Schwierigkeiten bereitet, soll er mittels Computersimulation geschätzt werden. Um die Ergebnisse aus diesem Simulationsexperiment mit einer vorgegebenen Genauigkeit erreichen zu können, ist eine Versuchsplanung erforderlich.

\subsection{Planung eines Laufes des Simulationsexperiments}

Es müssen die zu simulierenden Größen festgelegt werden. Das sind neben dem Grad der Balanciertheit der $\mathrm{zu}$ verwendenden Versuchspläne $\left(\mathrm{N}, \mathrm{a}, \mathrm{n}_{1}, \mathrm{n}_{2}, \ldots, \mathrm{n}_{\mathrm{a}}\right)$, die Verteilung der $\underline{u}_{i}$ und $\underline{e}_{i j}$ und das Verhältnis der beiden Varianzkomponenten $\sigma_{u}^{2} / \sigma_{e}^{2}$. Mit den Versuchsplänen soll begonnen werden.

Wir wählen $\mathrm{a}=10$ und $\mathrm{N}=\sum_{\mathrm{i}=1}^{10} \mathrm{n}_{\mathrm{i}}=100$ und wollen den Einfluß der Balanciertheit über die Pläne der folgenden Tabelle erfassen. 
Tabelle

Versuchspläne $\left(N=100 ; a ; n_{i}\right)$ für ausgewählte Simulationsuntersuchungen (Experimental designs $\left(N=100 ; a ; n_{i}\right)$ used for special simulation investigations)

\begin{tabular}{cccccccccccc}
\hline Versuchsplan & $\mathrm{a}$ & $\mathrm{n}_{1}$ & $\mathrm{n}_{2}$ & $\mathrm{n}_{3}$ & $\mathrm{n}_{4}$ & $\mathrm{n}_{5}$ & $\mathrm{n}_{6}$ & $\mathrm{n}_{7}$ & $\mathrm{n}_{8}$ & $\mathrm{n}_{9}$ & $\mathrm{n}_{10}$ \\
\hline 1 & 10 & 1 & 1 & 1 & 1 & 1 & 1 & 1 & 1 & 1 & 91 \\
2 & 10 & 2 & 2 & 2 & 2 & 2 & 2 & 2 & 2 & 2 & 82 \\
3 & 10 & 3 & 3 & 3 & 3 & 3 & 3 & 3 & 3 & 3 & 73 \\
4 & 10 & 4 & 4 & 4 & 4 & 4 & 4 & 4 & 4 & 4 & 64 \\
5 & 10 & 5 & 5 & 5 & 5 & 5 & 5 & 5 & 5 & 5 & 55 \\
6 & 10 & 6 & 6 & 6 & 6 & 6 & 6 & 6 & 6 & 6 & 46 \\
7 & 10 & 7 & 7 & 7 & 7 & 7 & 7 & 7 & 7 & 7 & 37 \\
8 & 10 & 8 & 8 & 8 & 8 & 8 & 8 & 8 & 8 & 8 & 28 \\
9 & 10 & 9 & 9 & 9 & 9 & 9 & 9 & 9 & 9 & 9 & 19 \\
10 & 10 & 10 & 10 & 10 & 10 & 10 & 10 & 10 & 10 & 10 & 10 \\
\hline
\end{tabular}

Weiterhin sollen die Verteilungen von $\underline{\underline{u}}_{\mathrm{i}}$ und $\underline{\mathrm{e}}_{\mathrm{ij}}$ über ihre Schiefe und Exzeß variiert werden. Um den praktisch interessanten Bereich von Schiefe und Exzeß abstecken zu können, nutzen wir das Ergebnis von NÜRNBERG (1980).

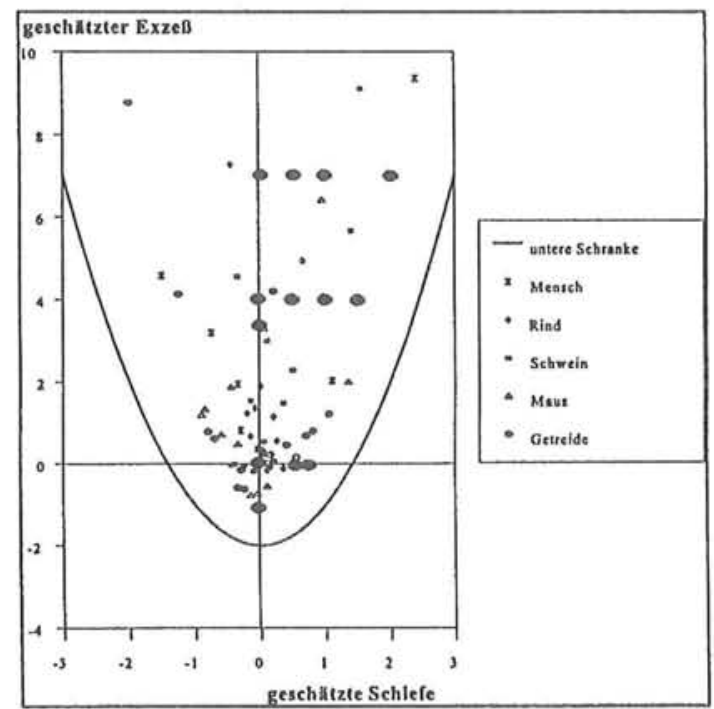

Abb 1: Geschätzte Schiefe und Exzeß der Verteilungen von unterschiedlichen Merkmalen bei Menschen, Tieren und Pflanzen (NÜRNBERG, 1980); simulierte Verteilungen für $\underline{u}$ und $\underline{e}$ - schwarze Punkte (Estimated skewness and kurtosis of distributions of different traits in men, animals and plants (NÜRNBERG, 1980); simulated distributions $\underline{\mu}$ and $\mathbf{e}$ - black dots)

Es gilt allgemein $\gamma_{2} \geq \gamma_{1}^{2}-2$.

Die Untersuchungen legen es nahe, $-1 \leq \gamma_{2} \leq 10$ und $0 \leq \gamma_{1} \leq 2$ zu wählen. Die dicken Punkte geben die gewählten Kombinationen von Schiefe und Exzeß bei der Simulation an. Jede Simulation kann nur ein Vertretersystem von Verteilungen nutzen. Hier wird das sogenannte Fleishmansystem verwendet. In jedem Punkt kann genau eine Vertei- 
lung mit vorgegebener Schiefe und vorgegebenem Exzeß gewählt werden. Natürlich gibt es aber in jedem Punkt sehr viele Verteilungen, die die Ergebnisse weiter variieren lassen. Dieses Problem haben alle Robustheitsuntersuchungen auf der Basis von Simulationen mit vorgegebenen Verteilungen.

Um den Einfluß der Größe der Varianzkomponente einschätzen zu können, setzen wir $\sigma_{\mathrm{u}}^{2}+\sigma_{\mathrm{e}}^{2}=1$ und geben $0 \leq \sigma_{\mathrm{u}}^{2} \leq 1$ vor.

Die Vorhersagegenauigkeit der BLUP wird mit Hilfe des MSE gemessen. Es gilt

$$
\operatorname{MSE}(\underline{\widetilde{u}})=E\left[(\underline{\tilde{u}}-\underline{u})^{\prime}(\underline{\tilde{u}}-\underline{u})\right]=\operatorname{Sp}[\mathrm{V}(\underline{\tilde{u}}-\underline{u})], d . h .
$$

als Beurteilungskriterium wird die Summe aller einzelnen MSE der Komponenten von ũ genutzt.

Im Simulationsexperiment wird der MSE der BLUP (im Ergebnisteil mit BLUP-SIM bezeichnet) und der Vorhersagen $\operatorname{EBLUP}\left(2,{ }^{*}\right)$ aus

$$
\overline{\mathrm{MSE}}=\frac{1}{\mathrm{~N}_{\mathrm{s}}} \sum_{\mathrm{k}=1}^{\mathrm{N}_{\mathrm{s}}} \mathrm{MSE}_{\mathrm{k}}
$$

geschätzt, wobei $\mathrm{N}_{s}$ die Anzahl der Simulationsläufe bezeichnet. Für $\mathrm{MSE}_{\mathrm{k}}$ gilt

$$
\operatorname{MSE}_{\mathrm{k}}=\sum_{\mathrm{i}=1}^{\mathrm{a}}\left(\mathrm{u}_{\mathrm{i}(\mathrm{k})}-\widetilde{\mathrm{u}}_{\mathrm{i}(\mathrm{k})}^{(1)}\right)^{2}
$$

mit $u_{i(k)}$ und $\widetilde{u}_{i(k)}^{(l)}$ aus dem k-ten Simulationslauf, wobei $\widetilde{u}_{i(k)}^{(1)}$ das Ergebnis des entsprechenden Vorhersageverfahrens $(1=0: \operatorname{BLUP} ; 1=1, \ldots, 4: \operatorname{EBLUP}(2,1))$ darstellt. Die Beschreibung der Güte eines Vorhersageverfahrens mittels MSE ist in der Regel das Werk eines Statistikers. Für den Züchter bezieht sich die eigentliche Frage der Güte eines derartigen Verfahrens jedoch immer auf den erreichbaren Zuchtfortschritt. Bezeichnet man mit u den Vektor der Realisierungen der Zuchtwerte in einem Simulationslauf, mit $\underline{\underline{u}}$ den Vektor für eine bestimmte Variante von BLUP bzw. $\operatorname{EBLUP}(2)$, so kann $(\mathrm{u}, \widetilde{\mathrm{u}})$ betrachtet werden. Selektiert man nun einen bestimmten Prozentsatz, so kann man $\Delta \mathrm{u}$ berechnen. $\Delta \mathrm{u}$ stellt den maximal möglichen Zuchtfortschritt dar. Bezeichnen wir mit $\Delta \mathrm{u}(\widetilde{\mathrm{u}})$ den Zuchtfortschritt in u, wenn nach $\tilde{u}$ selektiert wird, so kann damit die Wirksamkeit der Selektion nach u beurteilt werden. Die verschiedenen Varianten von EBLUP(2), aber auch andere Zuchtwertvorhersagen können auf dieser Grundlage verglichen werden. Dazu bildet man den Mittelwert der Selektionserfolge über alle Simulationsläufe.

\section{Planung des Umfangs des Simulationsexperiments}

Ein Simulationsexperiment muß auch vom Umfang her geplant werden. Der MSE oder auch der Zuchtfortschritt werden als Mittelwert geschätzt, daher kann die Versuchsplanung zur Schätzung eines Mittelwertes genutzt werden (Verfahren 3/21/0010 der Verfahrensbibliothek). Wir unterstellen, daß $\overline{\mathrm{MSE}}$ (Modell für (9)) eine nahezu erwartungstreue Schätzung des MSE darstellt, die hinreichend gut normalverteilt ist. Wegen der großen Anzahl von Wiederholungen ist der letzte Teil der Voraussetzung gesichert. Wir geben die Genauigkeit relativ vor und fordern, daß die halbe erwartete Breite $d$ des Konfidenzintervalls zu vorgegebenem $1-\alpha=0,952 \%$ 
der Standardabweichung $\sigma$ des geschätzten $\mathrm{MSE}_{\mathrm{k}}$ beträgt. Es gilt

$$
\mathrm{d}=\mathrm{u}_{1-\alpha / 2} \cdot \frac{\sigma}{\sqrt{\mathrm{n}}}
$$

und daraus folgt für $\alpha=0,05$ und $d=0,02 \cdot \sigma$

$$
n=\left(\frac{1,96}{0,02}\right)^{2}=9604 \text {. }
$$

Wir runden auf und wählen $\mathrm{N}_{\mathrm{s}}=10000$.

\subsection{Ergebnisse des Simulationsexperiments}

Die Simulationsergebnisse sind sehr umfangreich. Aus diesem Grunde werden hier nur die wesentlichen Ergebnisse mit Hilfe von Beispielen demonstriert.

\subsubsection{Die Abhängigkeit des MSE von der Balanciertheit}

Zunächst wird die Abhängigkeit des MSE von der Balanciertheit des Versuchsplanes demonstriert. Die Varianzkomponenten wählen wir $\sigma_{u}^{2}=0,175$ und $\sigma_{\mathrm{e}}^{2}=0,825$. Schiefe und Exzeß von $\underline{u}$ und $\underline{e}$ werden als Null angenommen (Normalverteilung wird vorausgesetzt). Das Ergebnis zeigt Abbildung 2.

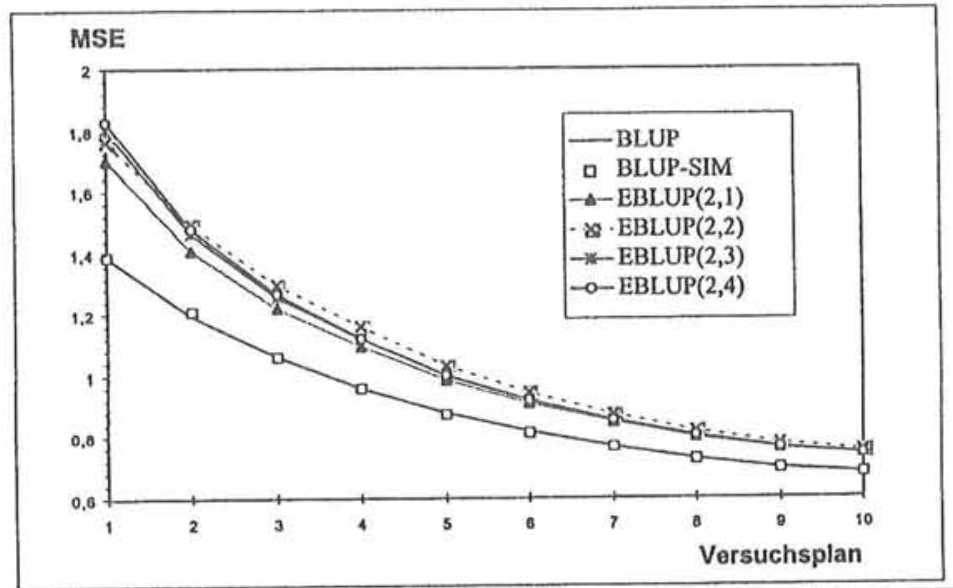

Abb. 2: Einfluß der (Un)balanciertheit der Versuchspläne auf den berechneten bzw. geschätzten MSE von BLUP bzw. der geschätzten BLUP - Normalverteilung, $\sigma_{\mathrm{u}}^{2}=0,175, \sigma_{\mathrm{c}}^{2}=0,825$ (Influence of the (un) balancedness of the experimental designs on the calculated and estimated MSE of BLUP and estimated BLUP normal distribution, $\sigma_{\mathrm{s}}^{2}=0,175, \sigma_{\mathrm{c}}^{2}=0,825$ )

Die erreichte Genauigkeit der Simulation wird durch die gute Übereinstimmung zwischen BLUP (MSE von BLUP berechnet) und BLUP-SIM (MSE von BLUP mittels Simulation geschätzt) demonstriert. Es ist auffällig, daß hier $\operatorname{EBLUP}(2,1)$ eigentlich bezüglich des MSE das beste Vorhersageverfahren darstellt. Analog sehen die Ergebnisse auch für andere Varianzkomponenten und Verteilungen mit Schiefe und Exzeß aus.

Der Einfluß der Balanciertheit des Versuchsplanes und der Schätzung der Varianzkom- 
ponenten auf den Selektionserfolg wird in Abbildung 3 demonstriert. Es wurde eine Selektionsintensität von $30 \%$ unterstellt. Hier erweisen sich $\operatorname{EBLUP}(2,3)$ und $\operatorname{EBLUP}(2,2)$ als die besten Verfahren.

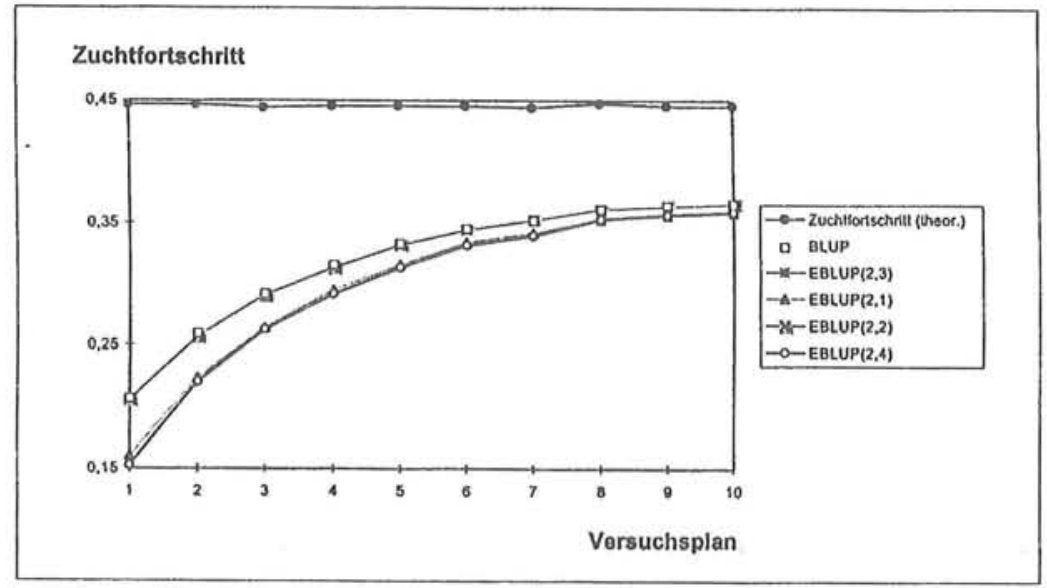

Abb. 3: Einfluß der (Un)balanciertheit der Versuchspläne auf den Zuchtfortschritt bei Selektion nach BLUP bzw. der geschätzten BLUP im Vergleich zum theoretischen Zuchtfortschritt - Normalverteilung, $\sigma^{2}=0,175$, $\sigma_{e}^{2}=0,825$, Selektionsintensität: $30 \%$ (Influence of the (un) balancedness of the experimental designs on genetic selection differential in the sense of selection by BLUP and estimated BLUP compared with the theoretic genetic selection differential - normal distribution, $\sigma_{\mathrm{u}}^{2}=0,175, \sigma_{\mathrm{e}}^{2}=0,825$, selection intensity: $30 \%$ )

\subsubsection{Die Abhängigkeit des MSE von den Varianzkomponenten}

Auch hier wird die Abhängigkeit nur für einen Versuchsplan, wir haben den Plan 4 gewählt, und für Normalverteilung demonstriert. Die Ergebnisse sind in Abbildung 4 dargestellt.

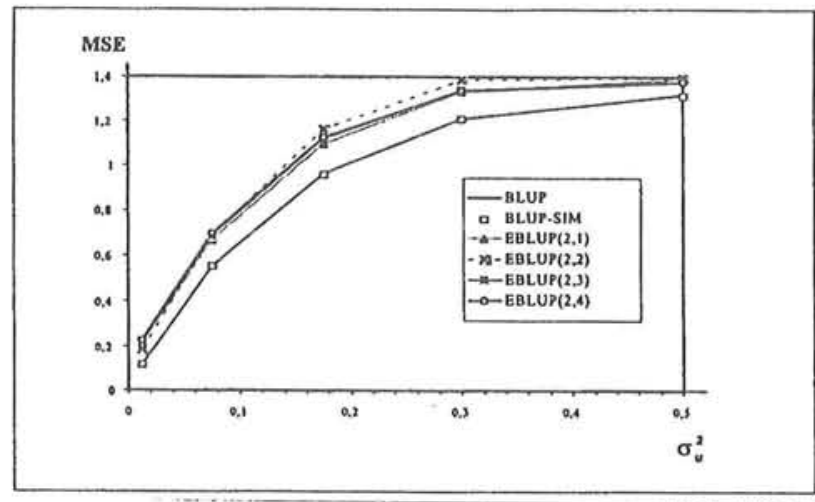

Abb. 4: Berechneter bzw. geschätzter MSE von BLUP bzw. geschätzter BLUP in Abhängigkeit von der Varianzkomponente $\sigma_{s}^{2}$ - Normalverteilung, Versuchsplan 4 (Tab.), $\sigma_{s}^{2}+\sigma_{c}^{2}=1$ (Calculated and estimated MSE of BLUP and estimated BLUP in dependence on the variance component $\sigma_{\mathrm{u}}^{2}$ - normal distribution, experimental design 4 (Tab.), $\sigma_{\mathrm{a}}^{2}+\sigma_{\mathrm{c}}^{2}=1$ ) 
Wieder ist EBLUP(2,1) das beste Verfahren.

Analoge Betrachtungen zum Zuchtfortschritt zeigen auch hier wieder eine Überlegenheit von $\operatorname{EBLUP}(2,2)$ und $\operatorname{EBLUP}(2,3)$, die sich außerdem nur sehr geringfügig von BLUP unterscheiden.

Die gemeinsame Abhängigkeit des MSE von BLUP von der Balanciertheit des Versuchsplanes (Tabelle) und $\sigma_{u}^{2}$ ist in Abbildung 5 dargestellt.

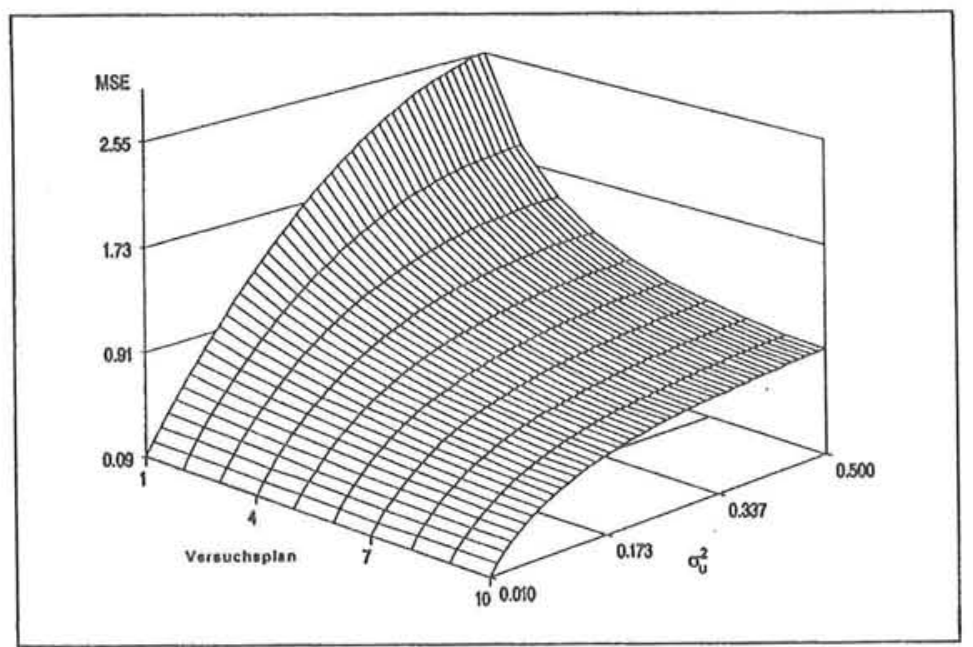

Abb. 5: Berechneter MSE von BLUP - Abhängigkeit von der Balanciertheit des Versuchsplanes und $\sigma_{*}^{2}$ (Calculated MSE of BLUP in dependence on the balancedness of experimental design and $\sigma_{\text {. }}^{2}$ )

Eine ähnliche Fläche auf höherem MSE-Niveau erhält man auch für die $\operatorname{EBLUP}\left(2,{ }^{*}\right)-$ Varianten, wobei sich der Niveauunterschied zwischen BLUP und EBLUP $\left(2,{ }^{*}\right)$ den Abbildungen 2 und 4 entsprechend gestaltet.

\subsubsection{Die Abhängigkeit des MSE von Schiefe und Exzeß}

Zusammenfassend kann festgestellt werden, daß die Schiefe der Verteilung keinen bemerkenswerten Einfluß auf den MSE besitzt.

Das wird an einem Beispiel in der Abbildung 6 belegt.

Der Exzeß der Verteilung hat einen Einfluß auf den MSE. Das wird an einem Beispiel demonstriert. Wir wählen die Schiefe jeweils Null und nutzen den Versuchsplan 4 und die Varianzkomponenten $\sigma_{\mathrm{u}}^{2}=0,175$ und $\sigma_{\mathrm{e}}^{2}=0,825$.

Im untersuchten Bereich von $-1 \leq \gamma_{2} \leq 7$ scheint die Abhängigkeit des MSE linear zu sein. Mit wachsendem Exzeß fällt der MSE. Insbesondere negative Werte des Exzeß vergrößern also den MSE. Sowohl Schiefe als auch Exzeß beeinflussen die Überlegenheit von EBLUP(2,1) jedoch nicht. Der Einfluß des Exzesses auf den MSE ist nur moderat. 


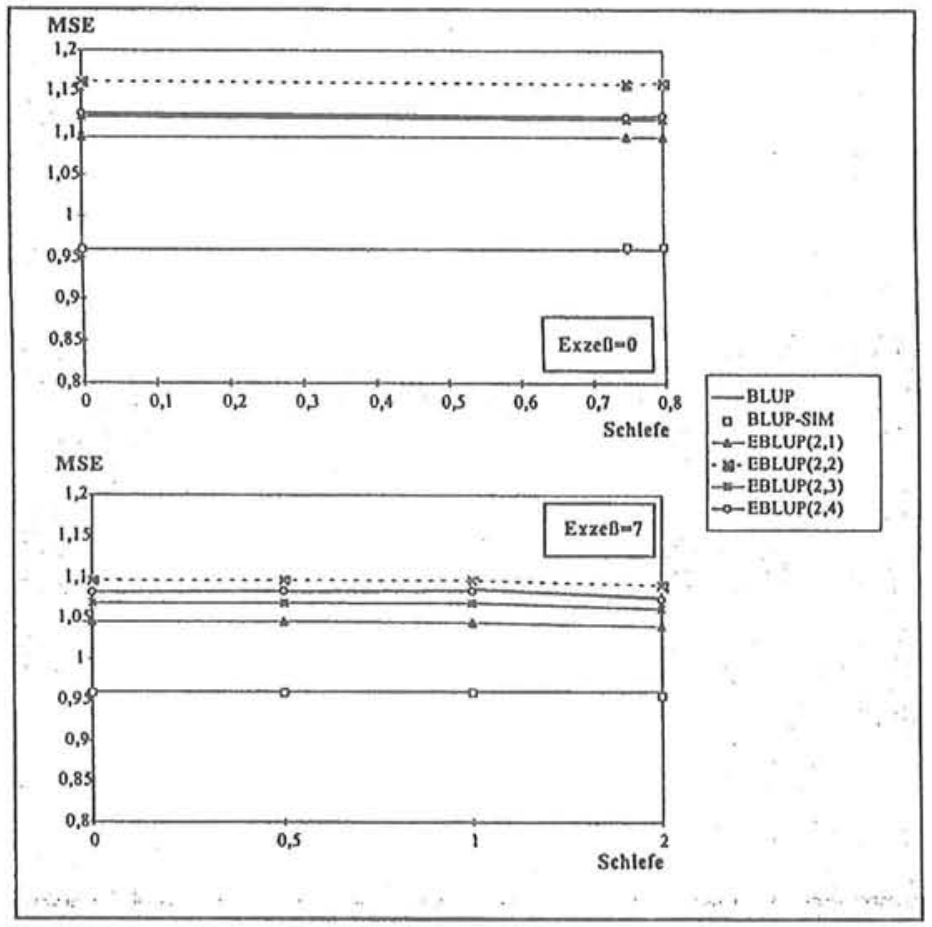

Abb. 6: Einfluß der Schiefe der Verteilungen von ㅆ und e auf den berechneten bzw. geschătzten MSE von BLUP bzw. der geschätzten BLUP - $\sigma_{,}^{2}=0,175, \sigma_{e}^{2}=0,825$, Versuchsplan 4 (Influence of skewness of the distribution of $\underline{\underline{u}}$ and $\underline{\mathrm{e}}$ on the calculated and estimated MSE of BLUP and estimated BLUP $-\sigma^{2}=0,175$, $\sigma_{\mathrm{e}}^{2}=0,825$, experimental design 4)

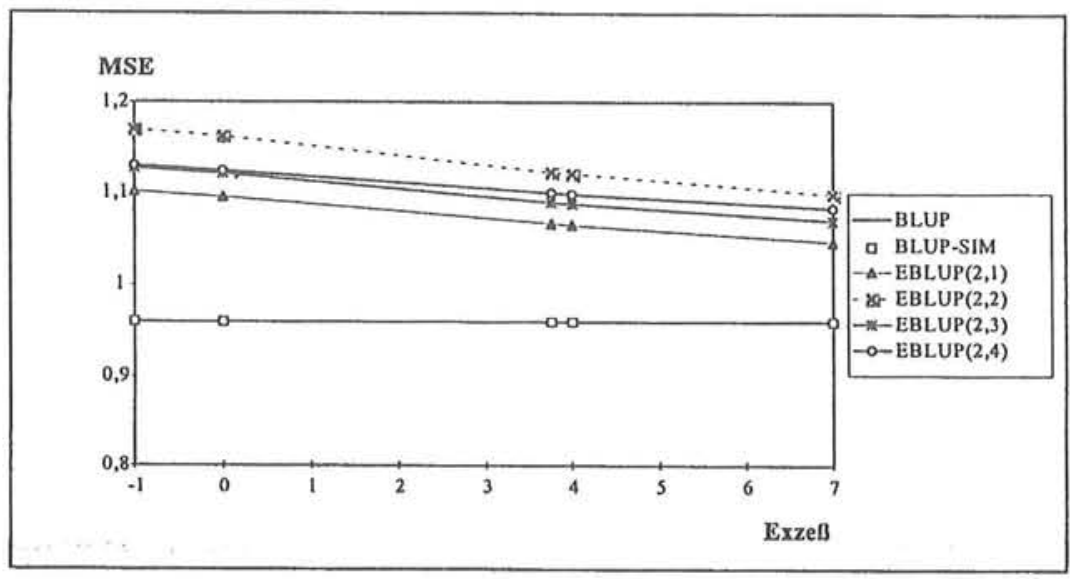

Abb. 7: Einfluß des Exzesses der gemeinsamen Verteilung von $\underline{\underline{u}}$ und $\mathrm{e}$ auf den berechneten bzw. den geschätzten MSE von BLUP bzw. der geschätzten BLUP - Schiefe $=0, \sigma_{4}^{2}=0,175, \sigma_{\text {, }}^{2}=0,825$ und Versuchsplan 4 (Influence of kurtosis of the distribution of $\underline{y}$ and $\underline{e}$ on the calculated and estimated MSE of BLUP and estimated BLUP - skewness $=0, \sigma_{\text {, }}^{2}=0,175, \sigma_{c}^{2}=0,825$, experimental design 4) 
5. Bemerkungen zur Berechnung des MSE von BLUP und EBLUP(2) mit SAS

Für das Modell von Abschnitt 4 hat der MSE von BLUP (8) bei bekannten Varianzkomponenten die Form

$$
\operatorname{MSE}(\underline{\tilde{u}})=\sigma_{u}^{2}\left(a+\sigma_{u}^{2}\left(\frac{\sum_{j=1}^{a}\left(\frac{n_{j}}{\sigma_{e}^{2}+n_{j} \sigma_{u}^{2}}\right)^{2}}{\sum_{j=1}^{a} \frac{n_{j}}{\sigma_{e}^{2}+n_{j} \sigma_{u}^{2}}}-\sum_{j=1}^{a} \frac{n_{j}}{\sigma_{e}^{2}+n_{j} \sigma_{u}^{2}}\right)\right)
$$

als Summe der Komponenten

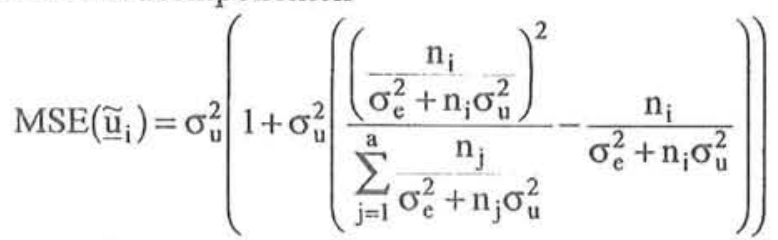

Als Genauigkeitsangabe für BLUP wird bei bekannten Varianzkomponenten von der Prozedur mixed in SAS völlig korrekt der Standardfehler der Vorhersage verwendet, welcher identisch mit der Wurzel von (12) ist. Problematischer wird es bei EBLUP(2). Wegen numerischer Schwierigkeiten zieht man sich auf die sogenannte naive Approximation des MSE zurück, die beinhaltet, daß in (12) die Varianzkomponenten durch entsprechende Schätzwerte, die aus den $y_{i j}$-Werten ermittelt wurden, ersetzt werden.

In der Abbildung 8 wird deutlich, daß man bei der Anwendung der naiven MSEApproximation vorsichtig sein muß. Der tatsächliche MSE der EBLUP(2) kann in der Tat erheblich größer sein, als mit dieser Approximation ausgewiesen.

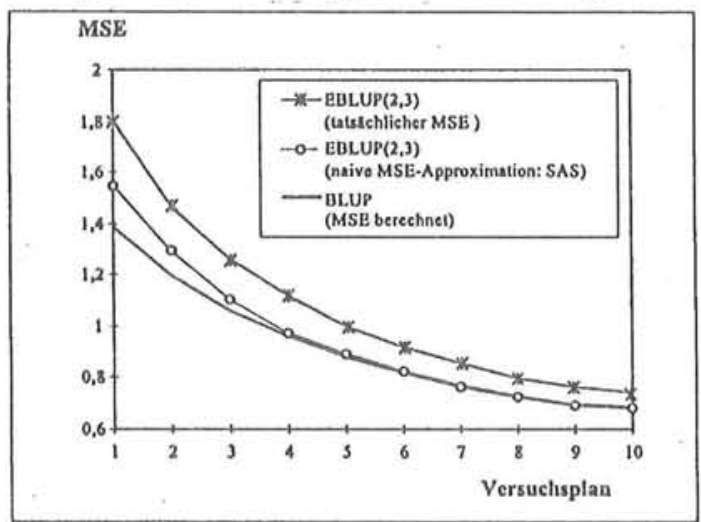

Abb. 8: Naive Approximation des MSE der EBLUP(2,3) im Vergleich zum tatsächlichen MSE der EBLUP(2,3) (mittels Simulation geschätzt) und zum berechneten MSE der BLUP in Abhängigkeit von der (Un)Balanciertheit des Versuchsplanes - Normalverteilung, $\sigma_{\mathrm{s}}^{2}=0,175, \sigma_{\mathrm{s}}^{2}=0,825$ (Naive MSE approximation of EBLUP $(2,3)$ in comparison with the real MSE of $\operatorname{EBLUP}(2,3)$ (estimated by simulation) and the calculated MSE of BLUP in dependence on the (un) balancedness of the experimental design - normal distribution $\sigma_{\mathrm{u}}^{2}=0,175, \sigma_{\mathrm{e}}^{2}=0,825$ ) 
Das hat zur Folge, daß gerade bei kleinen Versuchsumfängen erhebliche Überschätzungen der Genauigkeit der Vorhersage mit einer EBLUP(2)-Variante durch die naive MSE-Approximation auftreten können. Diese Genauigkeitsapproximation von EBLUP(2) sollte man erst bei hinreichend großem Gesamtversuchsumfang $N$ als $\mathrm{Ma} ß$ für die Vorhersagegenauigkeit verwenden.

Faßt man die Simulationsergebnisse zusammen, kommt man zu folgendem Fazit. In Abhängigkeit von den Modellparametern, vom Versuchsplan und vom Varianzkomponentenschätzer kann sich der MSE von EBLUP gegenüber dem MSE von BLUP zum Teil erheblich unterscheiden.

Der Unterschied zwischen den einzelnen Varianten der $\operatorname{EBLUP}\left(2,{ }^{*}\right)$ bezüglich der Varianzkomponentenschätzung war relativ moderat bei Schätzern, die Schätzwerte im Parameterraum liefern. Varianzkomponentenschätzer, die negative Schätzwerte liefern können (z.B. ANOVA), haben einen verheerenden Einfluß auf den MSE von EBLUP(2) und sollten aus diesem Grunde unbedingt vermieden werden. Von den verwendeten Varianzkomponentenschätzern schnitt bei dem einfachen Modell ANOVACOR am besten ab. Beim Bewertungskriterium 'Zuchtfortschritt' ist der Unterschied zwischen BLUP und EBLUP im Gegensatz zum MSE relativ gering, wenn man REML oder ML für die Schätzung der Varianzkomponenten verwendet. Das ist gerade für den Züchter das Entscheidende.

Generell kann man sagen, daß EBLUP eine brauchbare Methode zur Zuchtwertvorhersage ist und wegen der besseren Ergebnisse beim Zuchtfortschritt sollte man REML bzw. ML bei der Varianzkomponentenschätzung den Vorzug geben. Balanciertere Versuchspläne wirken sich positiv auf die Güte der Zuchtwertvorhersage aus. Zumindest lohnt es sich, darüber nachzudenken, inwieweit mit einer verbesserten Zuchtorganisation ('Versuchsplanung') die Zuchtwertvorhersage verbessert werden kann.

Der MSE von EBLUP(2) kann mittels Simulation hinreichend genau geschätzt werden. Die Genauigkeit dieser Schätzung ist über eine Versuchsplanung zum Simulationsumfang $\mathrm{N}_{\mathrm{S}}$ regelbar.

Die naive MSE-Approximation sollte nur bei hinreichend großem $\mathrm{N}$ angewendet werden.

\section{Literatur}

COCHRAN, W. G.:

Improvement by means of selection. Proc. second Berkeley Symp. Math. Stat. Prob. (1951) 449-470 EISENHART, C.:

The assumptions underlying the analysis of variance. Biometrics 3 (1947) 1-21

HENDERSON, C. R.:

Selection index and expected genetic advance. In: HANSON, W. D., ROBINSON, H. F.: Statistical genetics and plant breeding. National Academy of Sciences, Washington (1963) Publication 982, 141163

HENDERSON, C. R.:

Sire evaluation and genetic trens. In: Proceedings of the animal breeding and genetics symposium in honor of Dr. J. L. Lush. ASAS and ADSA, Champaign, Illinois (1973) 10-41 
HENDERSON, C. R.:

Best linear unbiased estimation and prediction under a selection model. Biometrics 31 (1975) 423-449

HERRENDÖFER, G.; TUCHSCHERER, A.:

HUBER, P.J.:

Selection and breeding. Journal of Statistical Planning and Inference vol. 54, N 3 (1996) $307-321$

Robust Statistics. John Wiley \& Sons, New York (1981)

NÜRNBERG, G.:

In: HERRENDÖRFER, G. (Federf.): Robustheit I. Probleme der angewandten Statistik, 4 (1980), FBN Dummerstorf

TUCHSCHERER, A.:

Some remarks on the prediction of breeding values by BLUP. Proceedings of the International Conference on Population Mathematics in Schwerin, Part II (1988) 120 - 133

TUCHȘCHERER, A.; HERRENDÖRFER, G.:

Mean estimators in the random one-way model. 1. overview on estimators with their necessary assumptions and properties of interest. Archiv f. Tierzucht 37 (1994) 1, 89-99

TUCHSCHERER, A.; RUDOLPH, P.E.; HERRENDÖRFER, G.:

Simulationsuntersuchungen zum Genauigkeitsverlust der besten linearen erwartungstreuen Vorhersage (BLUP) bei ủnbekannten Varianzkomponenten in gemischten linearen Modellen mit SAS. Proceedings der 1. Konferenz der SAS-Benutzer in Forschung und Entwicklung (KSFE) (1997) in Berlin 56 - 67

TUCHSCHERER, A.; HERRENDÖRFER, G.:

Evaluation of estimated BLUP in mixed linear models by a designed computer simulation. Proceedings of the 6th World Congress on Genetics Applied to Livestock Production, Armidale, INSW, Australia, (1998) 25: 585-588

TUCHSCHERER, A.; RUDOLPH, P.E.; TUCHSCHERER, M.; JÄGER, B.:

MSE-Schätzungen für die beste lineare erwartungstreue Vorhersage (BLUP) bei geschätzten Varianzkomponenten. Proceedings der 2. Konferenz der SAS-Benutzer in Forschung und Entwicklung (KSFE) (1998) in Jena, 288-302

TUCHSCHERER, A.; HERRENDÖRFER, G.; TUCHSCHERER, M.:

Evaluation of the best linear unbiased prediction in mixed linear models with estimated variance components by means of the MSE of prediction and the genetic selection differential. Biom. J. 40 (1998) 8,949-962

Eingegangen: 16.06 .1998

Akzeptiert: 15.12 .1998

Anschrift der Verfasser

Prof. Dr. GÜNTER HERRENDÖRFER, Dr. ARMIN TUCHSCHERER,

Dr. GERHARD DIETL, Dr. MARGRET TUCHSCHERER

Forschungsinstitut für die Biologie landwirtschaftlicher Nutztiere

Wilhelm-Stahl-Allee 2

D-18196 Dummerstorf 